\title{
Repenser la nation à partir du musée ? Le musée Julius Kambarage Nyerere en Tanzanie
}

\section{Marie-Aude Fouéré}

\section{Q OpenEdition}

\section{Journals}

\section{Édition électronique}

URL : https://journals.openedition.org/eastafrica/1509

DOI : 10.4000/eastafrica.1509

ISSN : 2790-1076

Éditeur

IFRA - Institut Français de Recherche en Afrique

\section{Référence électronique}

Marie-Aude Fouéré, «Repenser la nation à partir du musée ? Le musée Julius Kambarage Nyerere en Tanzanie », Les Cahiers d'Afrique de l'Est / The East African Review [En ligne], 56 | 2021, mis en ligne le 06 décembre 2021, consulté le 09 décembre 2021. URL : http://journals.openedition.org/eastafrica/1509 ; DOI : https://doi.org/10.4000/eastafrica.1509

Ce document a été généré automatiquement le 9 décembre 2021.

Les Cahiers d'Afrique de l'Est / The East African Review 


\title{
Repenser la nation à partir $\mathrm{du}$ musée ? Le musée Julius Kambarage Nyerere en Tanzanie
}

\author{
Marie-Aude Fouéré
}

«Dans les musées d'histoire contemporaine, les
portraits des pères de la nation ont remplacé
ceux des gouverneurs coloniaux, mais la plupart
continuent de fonctionner comme des lieux
d'autocélébration des pouvoirs en place.»
Anne Gauge (1999: 742).

1 Chaque année, dans le village de Butiama, à quelques kilomètres du lac Victoria, se tiennent les commémorations du décès du père de la nation tanzanienne, Julius Kambarage Nyerere. C'est à Butiama, dans le lieu-dit de Mwitongo, qu'est né et a grandi Nyerere ; c'est là qu'il est enterré. Le 14 octobre, jour de deuil national fait jour férié, le musée " Mwalimu ${ }^{1}$ Julius Kambarage Nyerere » ouvre grand ses portes pour accueillir des centaines de visiteurs. Ces célébrations sont l'occasion pour les pèlerins et les curieux, les politiciens et les élèves des écoles du pays de venir "apprendre » et se «rafrâichir la mémoire» sur les faits et gestes de celui qui a «apporté l'indépendance ", parfois « confirmer ce qui se lit dans les livres mais dont on veut voir des preuves tangibles », et surtout « honorer la mémoire du père de la nation $»^{2}$.

Dans ce musée entièrement dédié au premier président du Tanganyika (1961-1963) puis de la République unie de Tanzanie (1964-19853), l'œuvre politique nyérériste et l'histoire de la nation tanzanienne ne font qu'un. Suivant un processus classique de construction de la nation en Europe au XIXe siècle décrit par l'historien Ernest Renan (1997 [1882]), ce lieu a indéniablement pour vocation d'incarner l'idée nationale dans un grand homme et l'associer à un "passé héroïque » et glorieux ${ }^{4}$, celui de la lutte anticoloniale, de l'accès à l'indépendance, de la construction de l'État tanzanien et de l'ancrage d'un sentiment d'appartenance national. 
3 La raison d'être de ce musée patriotique, ouvert au public le 2 juillet 1999, paraît donc à première vue être affaire d'État ${ }^{5}$. Le baba wa taifa (père de la nation) constitue une ressource symbolique pour le pouvoir en place: depuis le décès de Nyerere en 1999, l'État-parti tente de perpétuer le grand roman national par le biais de la glorification de l'ancien président dans le but de revivifier le sentiment national ${ }^{6}$. Il s'agit simultanément de favoriser la longévité des élites politiques du parti au pouvoir depuis l'indépendance, le CCM (Chama Cha Mapinduzi, parti de la révolution ${ }^{7}$ ), et maintenir le jeu partisan fermé afin de bloquer toute alternance politique.

4 Cet article, cependant, ne vise pas simplement à dépeindre cette association classique, opérée par le haut, entre le culte des grandes figures politiques et les efforts étatiques de construction nationale, dont le cas tanzanien serait une illustration parmi d'autres. Il ambitionne d'utiliser le musée Nyerere comme un observatoire des compositions et recompositions dans les imaginaires des rapports entre gouvernants et gouvernés en Tanzanie contemporaine. Ce faisant, il questionne la valeur heuristique de cet objet, testant ses possibilités et pointant ses limites.

5 Certes, situé loin des grands centres du pays et des principaux axes de déplacement, le musée est faiblement fréquenté par les Tanzaniens. Ceux-ci, de manière générale, restent peu familiers des visites au musée ${ }^{8}$. Pourtant, ce qui se dit et s'écrit au moment des commémorations du décès de Nyerere dans ce site de déploiement délibéré de l'effort hégémonique étatique permet de s'interroger sur les acteurs et les processus de production des représentations politiques. En décryptant les pratiques de visite et les discours qui leur sont associés, on constate que l'investissement populaire contemporain dans la figure de Nyerere et à travers lui, dans l'idée nationale, est massif. Sachant que la figure de Nyerere n'a pas toujours été glorifiée, et ne l'est pas dans tous les milieux aujourd'hui, j'essaierai d'expliquer cet apparent consensus. En outre, je soulignerai que les manières de penser la nation ne sont pas de simples itérations des répertoires classiques du patriotisme tanzanien. Si elles continuent à s'appuyer sur l'ancien ethos socialiste d'éthique politique associant un modèle de vertu civique à des pratiques de redistribution des richesses, auquel est intimement associée la figure de Nyerere, elles laissent voir des déplacements de cette économie morale dans une séquence historique particulière, celle de transformations radicales dans les fonctionnements économiques, politiques et sociaux qui s'accompagnent d'une «démoralisation» (Ferguson, 2006) du politique en contexte postsocialiste (Fouéré, 2011 ; Mercer \& Jennings, 2011).

6 Ces remodelages dans les rapports imaginés entre gouvernants et gouvernés et les vertus qui les sous-tendent ne gagnent rien, toutefois, à être pensés suivant un paradigme binaire entre hégémonie et résistance. Au contraire, les resignifications actuelles du lien politique que les discours énoncés au musée Nyerere illustrent paraissent résulter d'ajustements récents, par petites touches, entre différentes catégories d'acteurs, qui ont abouti à forger un nouveau langage politique partagé à l'échelle nationale 9 . Les déplacements dans les lignes de force rhétoriques du projet national en Tanzanie contemporaine résultent donc des transactions qui s'effectuent entre les groupes au sein de l'État et les populations. Ces transactions se font simultanément, en Tanzanie, sur le mode de la «collaboration » et de la " complicité », de la « convivialité $»^{10}$ et sur celui de la coercition et la violence. Une telle approche du musée souligne les limites d'une recherche qui, uniquement centrée sur cet objet politique, finirait par en hypertrophier la performativité. Le musée Nyerere est avant 
tout une caisse de résonance de mouvements de fond dans l'énonciation du politique qui s'opèrent hors du musée, dans des espaces sociaux multiples et entrecroisés. L'efficacité de ces espaces dans l'internalisation des rapports réels et imaginés entre gouvernants et gouvernés doit beaucoup au contrôle que l'État exerce sur eux et à leur visibilité médiatique. Réinsérer la visite au musée, en tant que pratique localisée, dans une situation, dans une conjoncture, dans un présent, pour mieux en saisir les enjeux, est donc indispensable.

7 L'analyse développée dans cet article, bien que centrée sur une institution muséale et les usages qui en sont faits, ne fait pas écho aux débats les plus courants aujourd'hui en muséologie ou en anthropologie sur la représentation de l'Afrique dans les musées, qui portent sur la mise en scène d'une authenticité stéréotypée et la production d'une esthétisation essentialiste. Elle porte encore moins sur la question du marquage des musées d'histoire, d'art et de culture en Afrique par l'ancien pouvoir colonial, qui ferait de ces lieux une "affaire de Blancs» (Gaugue, 1999) que les publics africains préféreraient ignorer ou bouder. Il ne s'agit pas, enfin, de s'interroger sur la pertinence ou la légitimité de l'importation d'un modèle muséal européen à vocation patriotique en Afrique, ni sur les possibilités d'invention d'un modèle africain du musée - une tâche qu'on laissera aux acteurs du patrimoine. La réflexion menée renvoie, au contraire, aux vastes questionnements en sciences sociales sur les rapports entre lieux de mémoire et production du nationalisme, sur les mises en scène et les performances de la nation, sur la microphysique du tissage des liens entre gouvernants et gouvernés.

8 Par ailleurs, si le musée Nyerere fait office de guide à travers le paysage des recompositions du projet national tanzanien, il est impossible de saisir ses divers modes d'appropriation sans l'inclure dans un itinéraire de mémoire comprenant le mausolée de Nyerere, qui accueille sa tombe, situé dans le jardin du grand domaine familial. Pour les visiteurs, le déplacement à Butiama se réduit rarement à la visite du musée, mais prend la forme d'un pèlerinage mémoriel où se mêlent le sacré et le profane, le religieux et le politique, le lointain et le familier (Fouéré, 2011a). L'article explore donc les pratiques muséales et les lectures de la nation qui leur sont associées tenant compte des catégories de visiteurs, de leurs motifs, de leurs usages du musée et de leur rapport au passé national - en réinsérant ce que je nomme le «muséemausolée » de Nyerere dans un parcours de mémoire plus vaste et dans le temps bien particulier de la commémoration.

\section{Dispositif muséal et hors-musée}

9 Le musée a souvent été théorisé comme une technologie de pouvoir en tant qu'il appartient aux institutions de l'État. Fruit d'un ensemble de décisions prises par le haut dont la mise en place est déléguée à des agents de l'État, à savoir les autorités muséales et les professionnels des musées, le musée véhicule des représentations officielles qui sont matérialisées dans des supports de communications aussi vecteurs de discours (objets, photos, vidéos, etc.) - aussi appelés «expôts » - et sont figées dans des scénographies (Desvallées, 1976; Merleau-Ponty \& Ezrati, 2006). Ces représentations officielles peuvent avoir trait à l'appartenance à des communautés restreintes, comme dans les musées locaux ou ethnographiques, ou à des regroupements plus larges, telle la communauté nationale dans les musées patriotiques ou d'arts et d'histoire. La communication de ces représentations par l'intermédiaire du musée a pour raison 
d'être d'assurer, dans le domaine du symbolique, ce qui s'obtient ailleurs (mais plus difficilement et par un investissement financier et humain bien plus important) par le biais de la coercition, insidieuse ou violente : l'ordre social, c'est-à-dire non seulement un certain état stabilisé des rapports entre État et citoyens, mais aussi le tracé de la frontière entre ces deux entités par laquelle l'État s'institue, au plan des représentations, comme une entité séparée du reste du monde social (Mitchell, 1991).

Dans cette perspective politique du musée comme lieu où s'entremêlent savoir et pouvoir, inspirée des travaux de Michel Foucault, le musée est pensé comme un instrument de discipline, d'ordre et d'obéissance. Pour reprendre les termes de Tony Bennett, dont l'audacieuse comparaison entre le musée et la prison comme institutions disciplinaires a été l'objet de vifs débats, le musée exerce son contrôle "à travers un pouvoir qui place les individus derrière lui, aveuglés dans la complicité avec lui plutôt que par soumission à lui » (Bennet, 1995 ${ }^{11}$ ). L'ordonnancement dans le musée doit refléter, pour le pouvoir en place, l'ordonnancement de la vie sociale tel qu'il est visé. Le musée ne fait donc pas qu'offrir un assemblage de matérialités au regard du visiteur pour le plaisir du loisir et du savoir. Les expôts ne sont pas des curiosités assemblées sur la base d'une valeur qui leur serait intrinsèque, jugée digne de protection et de valorisation ${ }^{12}$, encore moins des objets "silencieux » : ils constituent des supports de signification. Leur sélection, leur classification et leur disposition dans l'espace du musée résultent d'une stratégie sémiotique délibérée. Les processus de production de sens y sont donc contrôlés au travers de protocoles éprouvés - ou, tout du moins, sous tentative de contrôle. Politique muséale et politique d'État tout court sont ainsi étroitement associées. La recherche hégémonique est au cœur des mises en scène du grand roman national au musée africain, comme elle l'a été en Europe du XIX $X^{e}$ siècle, où les objets étaient des éléments " représentatifs d'une œuvre, d'une culture, d'un grand homme, bref d'une partie de la communauté imaginaire en question » (Poulot, 2009 ${ }^{13}$ ).

11 Bien que dispositif de pouvoir, le musée n'est pourtant jamais un outil hégémonique achevé qui aboutirait, par la force du symbolique, à l'imposition de significations prédéfinies sur des sujets passifs ${ }^{14}$. Tout d'abord, on aurait tort de supposer la cohérence des idéologies produites par le haut: l'État est un "palimpseste de contradictions" (Lonsdale et Berman, 1979: 491) entre des formes d'autorités en compétition, chacune ayant ses «mythes de légitimité et ses principes d'allégeance » (Lonsdale, 1981 : 154) qui se superposent au fil du temps, les plus récentes s'inscrivant sur celles qui ne sont encore qu'à demi effacées. En second lieu, les canaux de diffusion des représentations de la nation et du pouvoir sont multiples et poreux : le musée n'est pas le produit d'un unique auteur, à supposer que cette unicité serait gage de cohérence interne, mais de plusieurs groupes ou réseaux d'intérêts, tantôt solidaires tantôt en compétition, pouvant promouvoir des objectifs distincts, si ce n'est antagonistes. Ces groupes ont des loyautés diverses envers le projet national et des compétences variables à traduire ce projet en un dispositif muséal monosémique. Enfin, le public du musée n'est pas une tabula rasa sur laquelle s'inscrirait en grosses lettres le métarécit national. Il est au contraire multiple, composé de groupes disparates au capital socioéconomique et scolaire varié, aux rapports différents à l'histoire et au présent de leur pays. La multiplicité des allégeances des citoyens à leur patrie et de leur investissement dans l'État - fonction de leurs dispositions individuelles, sociales et culturelles - explique que la visite du musée puisse donner lieu à des interprétations différenciées. Le sens n'est donc pas simplement projeté sur les visiteurs, mais déchiffré 
au travers d'une grille de lecture déjà-là qui permet à ces derniers de produire ce sens, se le réapproprier, voire le déplacer ou le subvertir en y injectant des significations alternatives - et en y révisant, si besoin est, leur grille de lecture (De Certeau, 1980 ${ }^{15}$ ).

Ainsi, c'est moins la métaphore de la machinerie disciplinaire toute-puissante qui, à mon sens, est la plus pertinente pour caractériser le musée que celle de la communication. Cette métaphore est classique aujourd'hui dans les études en muséologie : le musée est un média dont la fonction communicationnelle se trouve à l'intersection d'une intention signifiante, d'un dispositif tangible et de son interprétation (Davallon, 1992, 1999). Cette fonction communicationnelle est toujours imbriquée dans des relations de pouvoir historiquement construites, c'est-à-dire dans des références qui se situent hors du musée. Dans ce cadre, si le musée peut être conceptualisé comme une "zone de contact " à la fois physique et symbolique ou rhétorique, pour reprendre l'expression forgée par James Clifford (1997), c'est moins dans une veine optimiste faisant du musée un espace d'approches collaboratives de "partage de l'autorité » pouvant aboutir à une représentation pacifiée des différents récits de la nation en coprésence ${ }^{16}$, que suivant une démarche critique propre aux sciences sociales qui fait sienne l'étude des frictions qui traversent le musée et mobilisent des références hors-musée ${ }^{17}$.

Mais le musée n'est pas non plus le lieu du « grand refus » (Foucault, 1976 ; 1997) où le chercheur pourrait observer le déploiement d'une capacité d'action des dominés face à l'État et ses institutions d'autorités. Il donne plutôt à voir comment les discours nationaux sont reproduits à travers des relations complices et interdépendantes qui lient les différents niveaux et réseaux d'acteurs composant la société. En Tanzanie, ce type de relations historiquement fondées pendant toute la période socialiste sur des pratiques de cooptation et de clientélisme, plus que sur la mise en ordre et la surveillance autoritaire - bien que des pratiques autoritaires aient aussi été déployées par le régime tanzanien -, avaient la spécificité de mettre sous tutelle, pour mieux les imbriquer au cœur des réseaux de l'autorité étatique, les organisations intermédiaires (syndicats, mouvements de jeunes, de femmes, etc.) et de développer des langages politiques "cohésifs" (langage de la tradition, des relations familiales, de la redistribution, etc.) qui, énoncés dans une langue unique promue langue nationale à l'indépendance, le swahili, ont été un élément symbolique majeur dans la mise en place d'une "culture politique » et d'un sentiment national robuste (Martin, 1988; Fouéré, 2011b). L'absence de contestation politique ouverte qui a longtemps caractérisé le régime tanzanien en période socialiste, et a engendré des pratiques qualifiées de désaffection, de démobilisation voire de "dépolitisation" (Campbell, 1992), tient à l'entretien de relations d'« intimité » (Mbembe, 2001) liant groupes sociaux dominants et subordonnés. Cette intimité résulte certes d'une violence insidieuse et du quadrillage du parti unique, mais aussi de forts mécanismes inclusifs. Elle explique qu'un fort investissement populaire dans le projet national peut cohabiter avec des modes de contestation feutrés mais bien réels, mais aussi que les élites tanzaniennes peuvent s'emparer des registres du populaire (Askew, 2002 ; Edmondson, 2007), comme je vais le montrer. 


\section{Le musée Mwalimu Julius Kambarage Nyerere}

14 Comme les autres musées dédiés aux pères de la nation en Afrique, le musée Nyerere à Butiama est conçu comme un mémorial, « hommage rendu par la nation à l'homme qui a présidé aux destinées du pays » (Gaugue, $1997: 92$ ), à ses talents, son charisme, son autorité. Il n'est pas, néanmoins, un musée posthume. Julius Nyerere en a impulsé la construction afin de collecter, conserver et exposer en un même lieu un ensemble d'objets, de documents et de photos d'importance historique mais attachés à ses fonctions et son statut de chef d'État. Nyerere, décédé le 14 octobre 1999 à l'hôpital Saint Thomas de Londres, a survécu de peu au musée, ouvert le 2 juillet de la même année. Malgré la maladie qui l'affaiblit (il est atteint de leucémie et part se faire traiter en Grande-Bretagne début septembre 1999), il assiste à la cérémonie d'ouverture, présidée par l'ancien Premier ministre Frederick T. Sumaye. Le musée, construit au pied de la butte où se dresse la maison de famille des Nyerere, vaste demeure en pierre et aux larges baies vitrées dominant la plaine qui s'étend jusqu'au lac Victoria, possède le statut de musée national. Il est géré par l'institution du National Museum of Tanzania, un organisme gouvernemental regroupant les principaux musées du pays sous la tutelle du Département des Antiquités au sein du ministère des Ressources naturelles et du Tourisme ${ }^{18}$, mais aussi dépendant du ministère de la Culture. La brochure du musée, épinglée sous une vitrine à l'extérieur du musée, mais non disponible pour le visiteur les moyens financiers des musées n'étant pas à la hauteur de leurs ambitions - présente clairement les objectifs des autorités muséales: «Le National Museum of Tanzania reconnaît l'immense contribution du père de la nation Mwalimu Julius Kambarage Nyerere à la construction de l'unité nationale. (...) L'objectif de ce musée est de rappeler à chaque Tanzanien que l'unité nationale dont nous pouvons aujourd'hui nous enorgueillir est le produit du grand travail accompli par le fondateur de la nation Mwalimu J. K. Nyerere. ${ }^{19}$ La brochure porte de nombreux courts extraits des discours publics de Nyerere sur l'indépendance, l'unité, la solidarité. Il interpelle l'engagement patriotique de chacun : «(..) Tanzaniens d'aujourd'hui, comment participons-nous à préserver l'unité nationale dont nous avons hérité?» La finalité de vivifier le sentiment national par l'intermédiaire de la mise en scène de l'ancien président répond aux objectifs de la politique culturelle de la Tanzanie: "Le principal objectif de la Division de la Culture Nationale est d'aider au développement du nationalisme tanzanien et de la personnalité tanzanienne par la promotion des activités culturelles » (Mbughuni, 1974). Au prix de 1500 shillings tanzaniens par adulte, 500 par enfant (et 6500 pour les visiteurs étrangers $)^{20}$, le musée est très cher pour un paysan villageois, mais reste abordable pour un petit fonctionnaire ou un commerçant de la ville.

Le musée expose des items variés dans son architecture de case traditionnelle circulaire. La collection est présentée dans deux salles séparées par une cloison murale largement ouverte à ses deux extrémités. Les critères de la scénographie restent opaques tant les expôts semblent être disposés de manière désordonnée. On y trouve des photos légendées de la période de la lutte anticoloniale, de l'indépendance et des années de Nyerere à la tête de l'État, où celui-ci pose parfois seul mais souvent en compagnie de ses compagnons de lutte, de politiciens tanzaniens, ou de chefs d'État étrangers; des diplômes honoris causa ou des médailles remises par des institutions et universités étrangères; et des cadeaux offerts lors de ses tournées dans le pays ou à l'étranger, ceci à différents moments de sa trajectoire d'homme politique (photo 1). On 
y trouve notamment des objets reçus lorsqu'il a quitté le pouvoir : tabourets circulaires à trois pieds, coffres en bois, sculptures, pagnes portant son effigie, "habits traditionnels des différentes tribus ( $\mathrm{du}$ ) pays, arcs et lances et flèches de (leur) bravoure originelle, tuniques et peaux de panthères et de lions (des) chefs traditionnels $»^{21}$, boucliers en peau, couronnes, sceptres et autres regalia. Enfin, une vitrine présente quelques objets strictement personnels, principalement une radio TSF, une paire de souliers en cuir et un service à thé. Un large panneau au mur affiche une grande fresque biographique de Nyerere qui mêle informations personnelles et trajectoire politique.

Photo 1. Visiteurs à l'intérieur du musée Nyerere, 14 octobre 2011

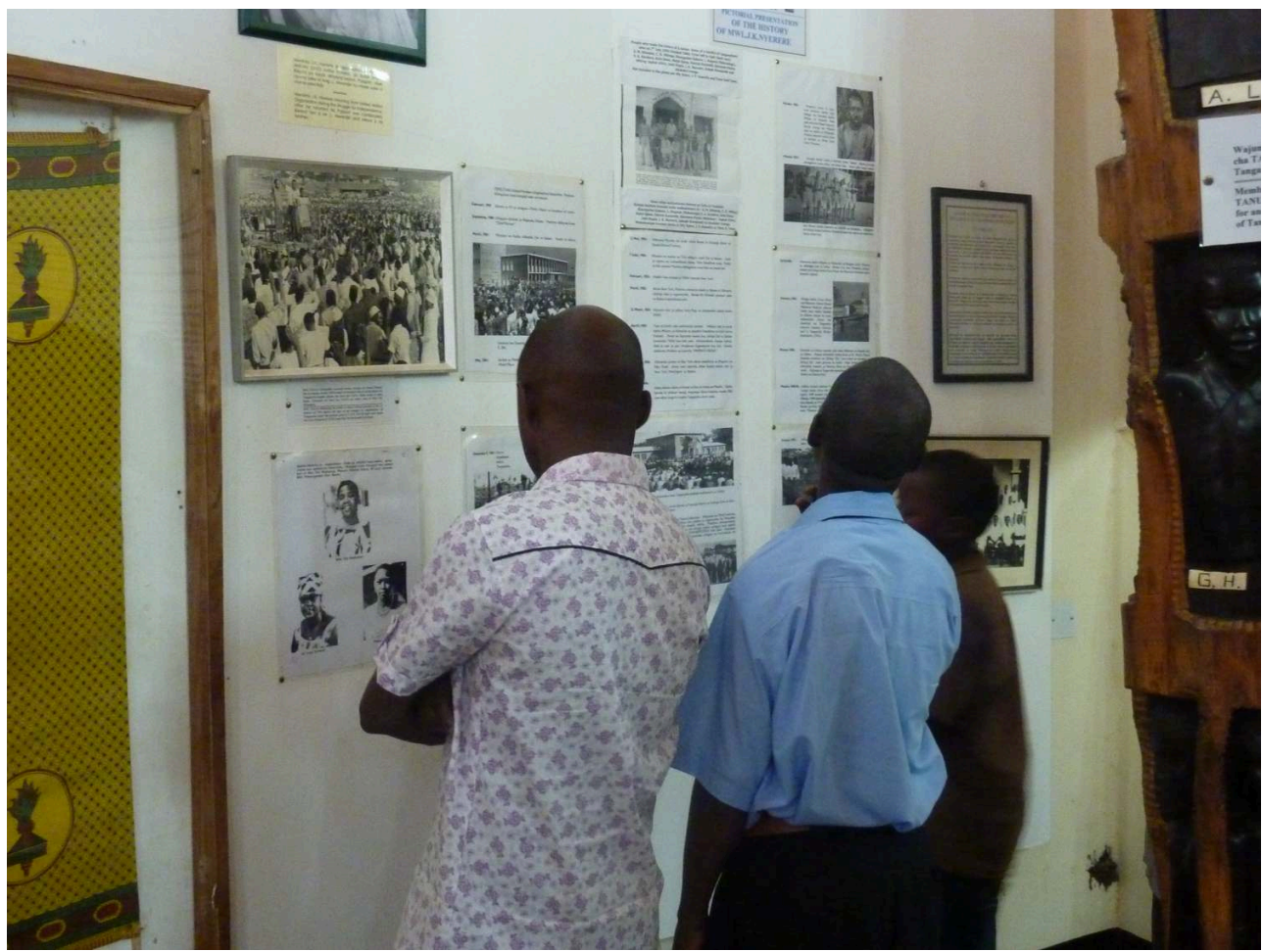

Photo : Marie-Aude Fouéré.

Dans une pièce située à l'entrée du musée, où le public signe à son arrivée le registre des visites, des vidéos des célèbres discours politiques de Nyerere sont rediffusées sur un écran télévision. Lors des commémorations de son décès, les clips de chants de lamentations en l'honneur de Nyerere passent en boucle. Ces chants, créés l'année de son décès et emplissant alors les ondes radiophoniques, "témoignaient d'une poussée du sentiment nationaliste après son décès, et offraient une évaluation laudative de ses contributions et de son héritage " (Askew, 2006). À ma visite en 2011, la chaîne publique TBC (Tanzanian Broadcasting Corporation) diffuse les chants de lamentations de groupes de musique fameux, parmi lesquelles celle de TOT (Tanzania One Theatre) intitulée " Nyerere, le leader du siècle »" ${ }^{22}$ de African Star Band, «Julius Nyerere », ou encore du célèbre musicien Remmy Ongala en association à quelques grands noms de la musique tanzanienne, dont de gros plans les montrent en pleurs, grimaçant de peine. C'est sous le regard de Nyerere portant l'habit traditionnel, dont un portrait-photo encadré et sous verre, à taille humaine, trône dans un des coins de la salle, que le 
visiteur redécouvre la voix de l'ancien président et entend celle de ses griots des temps modernes.

L'adhésion du personnel du musée à l'entreprise de mémorialisation de Nyerere transparaît tout autant dans les propos tenus au chercheur que dans un ensemble d'actions engagées pour diffuser la mémoire de l'ancien président. Le directeur du musée est un bon représentant de cette loyauté au projet national. C'est avec enthousiasme qu'il me rencontre pour la première fois en octobre 2011, lors de ma seconde visite au musée. Il me répète à l'envi que tout son personnel est à ma disposition, qu'on me donnera accès aux livres d'or et aux statistiques du nombre d'entrées, et que je suis autorisée à prendre autant de photos que je le souhaite. Après lui avoir signalé que j'avais publié différents articles universitaires sur Nyerere, il me relate sa présentation orale sur Nyerere lors d'un colloque dans une université tanzanienne où il est inscrit en $1^{\text {re }}$ année de doctorat, et affirme vouloir mettre par écrit ses réflexions sur la philosophie politique de Nyerere $^{23}$. Le directeur du musée est aussi très engagé dans la diffusion des idéaux de Nyerere auprès du public du musée, notamment auprès du public scolaire. L'après-midi de débats sur les politiques éducatives de Tanzanie organisée par le directeur du musée et rassemblant élèves et professeurs du secondaire, à laquelle j'assiste en octobre 2012, témoigne de ce fort engagement personnel à transmettre le legs moral et intellectuel du baba wa taifa. Chez un des responsables du musée, l'investissement nyérériste paraît même s'être incarné dans ses techniques du corps ${ }^{24}$.

La collection du musée est aussi objet de fierté chez le personnel du musée, qui organise la visite en débutant par les photos et objets historiques avant de passer aux cadeaux offerts à Nyerere, pour finir avec les affaires personnelles de Nyerere présentées avec émotion. Le musée ne rend pas seulement tangible l'itinéraire politique d'un homme et le cours de l'histoire nationale, mais génère des affects positifs envers Nyerere. Certes présentés sous une vitrine avec des vignettes très succinctes («les chaussures en cuir de Nyerere ", " tasse à thé et théière ", " la radio de Nyerere »), de tels objets de la sphère privée créent des effets de familiarité et d'intimité. En 2012, dès mon arrivée, les membres du personnel du musée me signalent, tout à tour, que le musée expose pour plusieurs semaines la torche de l'indépendance empruntée au musée de la Déclaration d'Arusha ${ }^{25}$. La flamme de cette torche, emblème de l'indépendance nouvellement acquise du Tanganyika en 1961, devait «briller au-delà de nos frontières, donnant espoir où se trouvait le désespoir, l'amour où se trouvait la haine, et la dignité là où avant il y avait seulement l'humiliation ${ }^{26}$. Elle fut allumée le jour de l'indépendance au sommet du Kilimandjaro par un officier de l'armée nationale, le lieutenant Alexander Nyirenda, qui y planta aussi le nouveau drapeau national. Depuis lors, une course de la torche est organisée annuellement, traversant plusieurs régions du pays avant d'être allumée chaque 9 décembre. Pendant toute la période nyérériste, elle fut constamment mobilisée comme objet symbolique, «signe de puissance et de pouvoir, de savoir aussi », placée sur les monuments, et montée plusieurs fois au sommet du mont Kilimandjaro (Martin, 1988 : 266).

19 Tous les expôts du musée Nyerere, loin donc d'être des objets silencieux, sont des vecteurs du grand roman national associé à la figure de Nyerere. Mais c'est autant par ce que le dispositif muséal montre que par ce qu'il ne montre pas, par la mise en scène que par la mise à l'écart, qu'une histoire sans accrocs de la construction nationale, toute entièrement attribuée aux talents de Nyerere, est déroulée sous le regard du 
visiteur. Le rôle des autres acteurs politiques, individuels ou collectifs (les associations, les premiers partis politiques, les syndicats, les mouvements de femmes, etc.), est gommé du musée. Les tensions au sein du parti et du gouvernement qui ont accompagné le régime de Nyerere, et ont abouti à l'emprisonnement ou l'exil de politiciens, sont ignorées. Et les épisodes qui ont durement touché la population et ont soulevé de fortes résistances, telle la campagne de villagisation qui prit une tournure coercitive au début des années 1972, sont également absents. L'« oubli » voire « l'erreur historique " (Renan, 1997 [1882]), ici comme ailleurs, participe à construire Nyerere comme un primus inter pares ayant seul présidé à la destinée du pays (Ranger, 1971; Fouéré, 2015a).

\section{Visiter le musée Nyerere}

Entre 2003 et 2011, les statistiques montrent que le musée est de plus en plus visité au fil des ans, toutes catégories confondues (2003: 4294 personnes; 2004: $4948 ; 2005$ : $5875 ; 2006: 6935 ; 2007: 7340 ; 2008: 6142 ; 2009: 9690$; entre octobre 2010 et octobre $\left.2011: 9443^{27}\right)$. C'est au mois d'octobre qu'il accueille le plus de visiteurs, un peu plus de 3000 pour l'année 2009 par exemple, alors que la moyenne mensuelle sur cette même année se monte à 807 visiteurs. Le mois d'avril, lorsqu'est célébrée l'Union entre le Tanganyika et Zanzibar (1964), est aussi une importante période de visites, avec 995 visiteurs en 2009. Ceci témoigne de l'association qui est faite, chez les visiteurs, entre Nyerere et la nation au moment des grandes fêtes nationales. Ces statistiques, qui distinguent les catégories "adultes", "scolaires» et "enfants", ainsi que "Tanzaniens » et «étrangers ", permettent aussi de noter la présence importante des écoliers - ils visitent le musée dans le cadre de sorties scolaires - ainsi que la faiblesse $\mathrm{du}$ nombre de visiteurs non-nationaux (environ 300). Elles ne nous disent rien, cependant, sur les profils socioéconomiques et professionnels des visiteurs, les raisons de leur visite, et leur perception du musée. Les livres d'or, les entretiens, les questionnaires et l'observation participante sont des outils plus adéquats pour cerner au plus près les attentes, les imaginaires et les effets de la visite au musée Nyerere.

Pour Consolata, qui se rend pour la première fois à Butiama en 2011, la visite de Butiama est conçue comme une démarche civique et patriotique, mais aussi comme un moment chargé d'émotions. Venue, en tant qu'agent du ministère de la Culture, des Sports, de la Communication et de la Jeunesse qui organise cette année-là les cérémonies du cinquantenaire de l'indépendance du Tanganyika à Butiama, elle se réjouit de pouvoir enfin découvrir le lieu de naissance et d'enterrement de celui qu'elle évoque d'emblée comme le père nourricier (baba mlezi) - reprenant à son compte les langages du paternalisme d'État - lors de notre trajet en voiture entre Musoma ${ }^{28}$, la ville du lac Victoria la plus proche de Butiama, située à une vingtaine de kilomètres. Consolata, qui se projette dans la découverte du site, est prolixe en anecdotes révélant son fort investissement affectif dans Nyerere. Elle évoque d'entrée de jeu le décès de Nyerere, rapportant que toute sa famille avait pleuré à chaudes larmes devant le poste de télévision, lors de la retransmission en direct de l'enterrement de Nyerere à Butiama le 23 octobre 1999. Elle insiste: "Même à l'enterrement de mon propre père, on ne pleurait pas autant ». Elle narre aussi cette anecdote maintes fois lue et entendue que les chefs d'État étrangers qui rendaient visite à Nyerere, lors de ses retraites loin de Dar es Salaam, s'étonnaient qu'un homme de cette stature réside dans un hameau reculé 
mal desservi. L'autre grand trope nationaliste, le swahili fait langue nationale pour favoriser l'unité de la nation, considéré comme l'œuvre de Nyerere, est aussi mis en avant.

Arrivée à Butiama, tandis que nous visitons la propriété familiale de Nyerere, Consolata rappelle, face à la statue d'une Vierge fixée au sommet d'un rocher que notre guide affirme avoir été un important lieu de prière pour Nyerere, que l'ancien président ne manquait pas ses messes quotidiennes même lorsqu'il était absorbé par les affaires de l'État à Dar es Salaam - un poncif que les médias nationaux entretiennent à chaque commémoration $^{29}$. Après avoir visité la maison de famille et s'être recueillie au mausolée, sur la tombe de Nyerere, Consolata prend le temps d'explorer le musée (photo 2). Elle s'émeut d'y trouver la série d'affaires personnelles de Nyerere exposées dans la vitrine contenant des chaussures, une radio et un service à thé. Pour mon interlocutrice, la découverte de Butiama est visiblement médiatisée par tout un ensemble d'informations en circulation dans l'espace public dont elle est détentrice. Elle lit et vit sa visite au travers de cette grille informative, de ce hors-musée qu'elle importe dans le musée et tout l'espace environnant, redécouvrant donc plus que découvrant, mais restant avide d'accumuler des détails qu'elle ne connaissait pas encore, et toute enjouée d'être en contact avec un espace imprégné de la présence de Nyerere.

Photo 2. Couple faisant prendre leurs deux jeunes enfants en photo assis sur la tombe de Nyerere à l'intérieur du mausolée, 14 octobre 2011

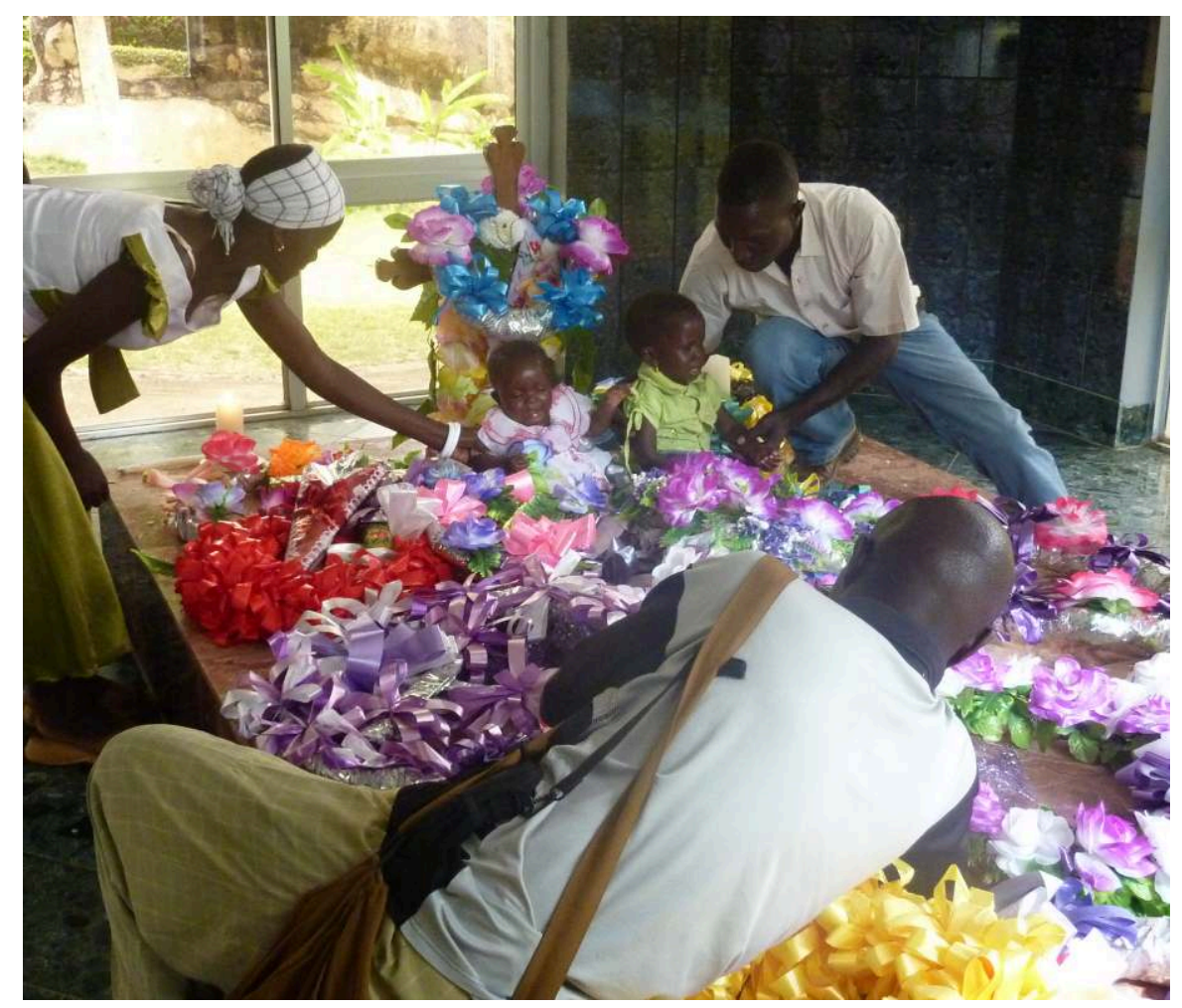

Photo : Marie-Aude Fouéré.

Dans son allégeance au patriotisme nyérériste, Consolata est représentative de la catégorie des agents de l'État qui, étant des trentenaires ou des quarantenaires ayant grandi à l'ombre de l'héritage du père de la nation, éduqués et lecteurs de la presse ou 
d'ouvrages actuellement acquis à la glorification de Nyerere, et généralement affiliés au parti au pouvoir en raison de leurs activités professionnelles, perpétuent une mémoire nostalgique de Nyerere. Consolata affiche ostensiblement cette loyauté à l'idée nationale arrimée à un Nyerere romancé. Assises toutes deux sur une grande natte protégée par un auvent, nous assistons, ce même 13 octobre, aux répétitions des parades des commémorations pour le cinquantenaire de l'indépendance du Tanganyika qui se tiennent officiellement le lendemain. Lorsque l'hymne national retentit, Consolata s'offusque que le public rassemblé, principalement composé des mères des écoliers mobilisés pour les parades, ne se lève ni ne reprenne l'hymne. Elle se lève soudainement et répète avec emphase: "C'est une honte, c'est une honte!", entonnant seule l'hymne au milieu des femmes restées assises et muettes. Toutefois, on rappelle qu'au sein de ce groupe social des fonctionnaires d'État, le sentiment d'être orphelin de Nyerere n'est pas nécessairement attaché à un regret pour un socialisme d'État disparu, tant les positions acquises des classes moyennes d'aujourd'hui sont le produit de la sortie du socialisme (Askew, 2006 ; Fouéré, 2011b). Par ailleurs, la critique envers la figure de Nyerere se manifeste plus visiblement dans les espaces et les milieux marginalisés au plan politique et économique (Becker 2013 ; Fouéré, 2013), ou au sein d'une nouvelle génération de classes moyennes désireuses d'entrer de plain-pied dans l'économie de marché.

Dans les commentaires des livres d'or du musée (2008-2012 $2^{30}$ mais aussi dans les questionnaires distribués après la visite en 2012 transparaît un même attachement à Nyerere, autant à l'homme et à ses qualités humaines qu'au chef de l'État et à ses accomplissements politiques. On vient ainsi en visite à Butiama honorer la mémoire de ce "héros", de ce "grand homme", du "père de la nation », et prier pour "qu'il repose en paix ». Certaines remarques, éloquentes, semblent une directe transposition de la rhétorique étatique associée à Nyerere : « La contribution de Mwalimu à la nation est incommensurable, il s'est vraiment donné pour la nation» (02/09/2009); «Il a œuvré pour sa nation et son peuple pour le bénéfice de la nation» $(28 / 07 / 2011)$. Beaucoup louent le musée de conserver et transmettre son souvenir « aux générations futures ». Chez beaucoup, la visite du musée ravive indéniablement la fibre patriotique : «La Tanzanie est unie et doit le rester » (16/05/2011). Le potentiel éducatif du musée revient fréquemment dans les commentaires qui soulignent que la visite a permis de renforcer la connaissance de la vie de Nyerere et de l'histoire du pays, voire tout simplement de les découvrir. À cet égard, certains recommandent que les écoliers et les lycéens viennent en nombre visiter le musée. Néanmoins, il est souligné à de multiples reprises que le site devrait se faire mieux connaître et être moins coûteux afin d'être accessible à tous. Les avis critiques sur la qualité du musée renvoient à l'envie d'en savoir plus, ou de mieux protéger ce savoir - même s'il est parfois souligné que le musée n'est pas à la hauteur de celui qu'il est censé (re)présenter : il faudrait plus de photos et les laminer afin qu'elles soient mieux conservées ; les livres de Nyerere et sur Nyerere, les CD de ses discours devraient être mis en accès libre dans le musée et disponibles à la vente; l'épisode de la maladie de Nyerere avant son décès est fâcheusement absent; la liste de ses plats préférés devrait figurer, etc. Ces appels à favoriser des rapports de familiarité voire d'intimité à Nyerere, par le biais du dispositif muséal et de ses expôts, sont récurrents. Ils s'expriment aussi dans quelques demandes écrites de rencontrer Maria Nyerere, la veuve de Julius Nyerere. Cette dernière réside dans la maison adjacente au musée, ouvre au public son jardin chaque 14 octobre, offre 
un repas gratuit aux villageois et aux visiteurs, et se rend souvent disponible pour des interviews.

Il est frappant de constater que, dans le corpus de commentaires recueillis, aucun avis dépréciatif sur Nyerere ou sur les politiques mises en place sous sa présidence n'a été formulé. Plutôt que d'attribuer ce fait à l'efficace d'un dispositif muséal qui serait une grande machinerie symbolique de persuasion, je voudrais faire l'hypothèse qu'entreprendre une visite à Butiama est gage d'un investissement préalable dans la figure de Nyerere, de même que l'est la pratique consistant à jeter quelques phrases sur les pages du livre d'or. Dans un pays où les réflexes d'autocensure sont encore en place, laisser la trace écrite - et nominative - d'opinions adverses au métarécit étatique requiert aussi un caractère frondeur peu commun.

La critique, lorsqu'elle apparait, porte sur les dirigeants politiques d'aujourd'hui : "Nous demandons à nos dirigeants qu'ils honorent feu Mwalimu Julius pour tout ce qu'il a fait pour notre nation au lieu de s'adonner à la prédation et la corruption " (09/04/2012) ; «Si Mwalimu ressuscitait et voyait une gestion des affaires publiques si mauvaise et corrompue aujourd'hui, il ne pourrait pas respirer l'air de la Tanzanie plus d'un quart de seconde » (22/06/2012). Ces remarques s'ancrent dans la construction idéalisée de la figure de Nyerere par le régime en place depuis son décès, après une période post-Ujamaa, entre 1985 et 1999, où il semblait peu certain que le pouvoir en place mise à long terme sur un legs moral nyérériste (Fouéré, 2015). Mais ces commentaires prennent aussi sens dans la configuration actuelle où Nyerere a été promu comme un étalon de mesure de la moralité politique en raison de la visibilité de la grande corruption associée au creusement du fossé entre les plus riches et les plus pauvres. La récurrence du terme swahili ufisadi dans les livres d'or, maintenant institué pour dénoncer la collusion entre la finance et la politique, laisse voir combien les notions les plus médiatiques deviennent des slogans dont le langage courant s'empare. Ainsi, la dénonciation de la démoralisation de la vie politique que l'on trouve dans les livres d'or n'est pas un effet de la visite: c'est bien plus la visite qui active des répertoires de contestation politique en circulation dans l'espace public - montrant que le musée est une caisse de résonance de dynamiques situées hors de lui. Ces répertoires de la contestation par lesquels Nyerere en est venu à constituer un étalon de mesure de la vertu politique ont été constitués par itérations constantes entre les protestations populaires contre l'immoralité des dirigeants d'aujourd'hui et les promesses des hommes politiques de marcher dans les pas du Mwalimu (Fouéré, 2015 ; Paget, 2020). Ironiquement, le tombeau, le musée Nyerere sont devenus des passages obligés pour les hommes politiques qui promettent d'être à la hauteur de l'incorruptibilité dont Nyerere fit preuve de son vivant (photo 3). 
Photo 3. Visite et interview d'une politicienne du CCM à Butiama, 14 octobre 2020

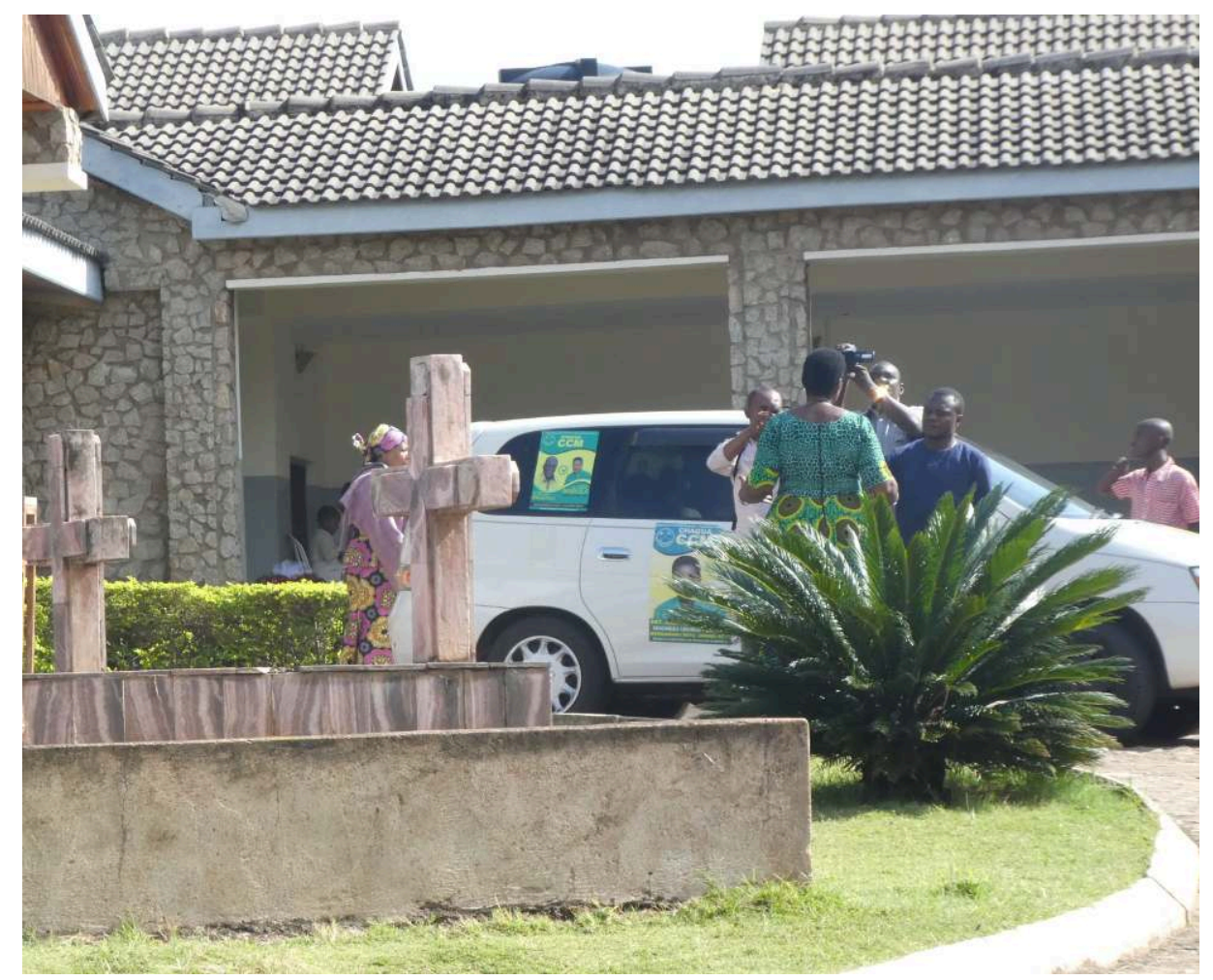

Photo : Marie-Aude Fouéré.

\section{Conclusion}

Prendre le musée pour objet politique, c'est chercher le politique en dehors des lieux institutionnalisés de l'expression politique - une approche aujourd'hui classique dans les travaux inspirés de la science politique par le bas (Martin, 1989). Mais c'est aussi chercher le politique dans le quotidien et le minuscule de pratiques sociales qui ne reposent sur aucun programme politique articulé, loin notamment de l'effervescence de l'action collective protestataire où se cherche le plus souvent, dans les études sur les rapports entre dirigeants et dirigés sur le continent africain, l'expression du dissentiment, si ce n'est de la contestation politique. L'article a voulu montrer, dans le cas d'un musée patriotique où l'intention hégémonique est manifeste, que la lecture qui en est faite par les visiteurs - politiciens en recherche de légitimité exclus - renvoie aux luttes idéologiques en cours. C'est justement parce que le musée Nyerere est un monument à la gloire des catégories du juste, du moral, du don de soi pour la nation telles qu'elles ont été promues par le passé - et dont Nyerere est devenu l'allégorie qu'il active, chez ses visiteurs, un mécanisme de mise en regard des rapports État et citoyens entre hier et aujourd'hui. On ne peut comprendre les lectures morales du musée que font les visiteurs hors d'un horizon d'attentes, ici déçues, de redistribution matérielle que l'État tanzanien a échoué à mettre en place depuis les années 1990. C'est tout un ethos économique fondé sur un modèle paternaliste et protecteur dans lequel le régime devait subvenir aux besoins du peuple que l'après-socialisme a ébranlé.

L'observation attentive des pratiques de visite au musée Nyerere dévoile aussi un rapport pacifié, convivial du processus de création des sujets politiques en Tanzanie. ${ }^{31}$ 
Nyerere fait l'objet d'un investissement affectif fort - bien que variable selon les milieux considérés - que laissent entrevoir, par exemple, les séances de photos prises par les visiteurs devant les photographies exposées ou les portraits peints de Nyerere exposés dans le musée. L'image du "père» (baba) de la nation tanzanienne, incessamment employée, témoigne de ces frictions entre, d'un côté, la crainte et le respect, mais aussi, de l'autre, la familiarité et l'intimité. Mais parce que Nyerere est coextensif avec l'idée de la nation tanzanienne, c'est bien une conception de l'appartenance nationale chargée des affects du familier qui se donne à voir dans les attitudes et les paroles des visiteurs, conception non pas uniquement fruit d'une « imagination muséifiante» (Anderson, $1995: 181$ ) stato-centrée mais aussi résultat d'un nationalisme banal et quotidien (Billig, 1995). C'est cette convivialité ordinaire qui rend possible des comportements légers et des attitudes badines dans un espace de mémoire pourtant marqué par une dramaturgie grave et solennelle. En octobre 2012, un jeune couple enlacé se faisait prendre en photo, tout en jeans moulants et couleurs pétillantes (photo 4). Cette banalisation de la visite au musée Nyerere traduit un sentiment de conscience national toujours robuste et amène.

Photo 4. Jeune couple se faisant prendre en photo à Butiama, 14 octobre 2012

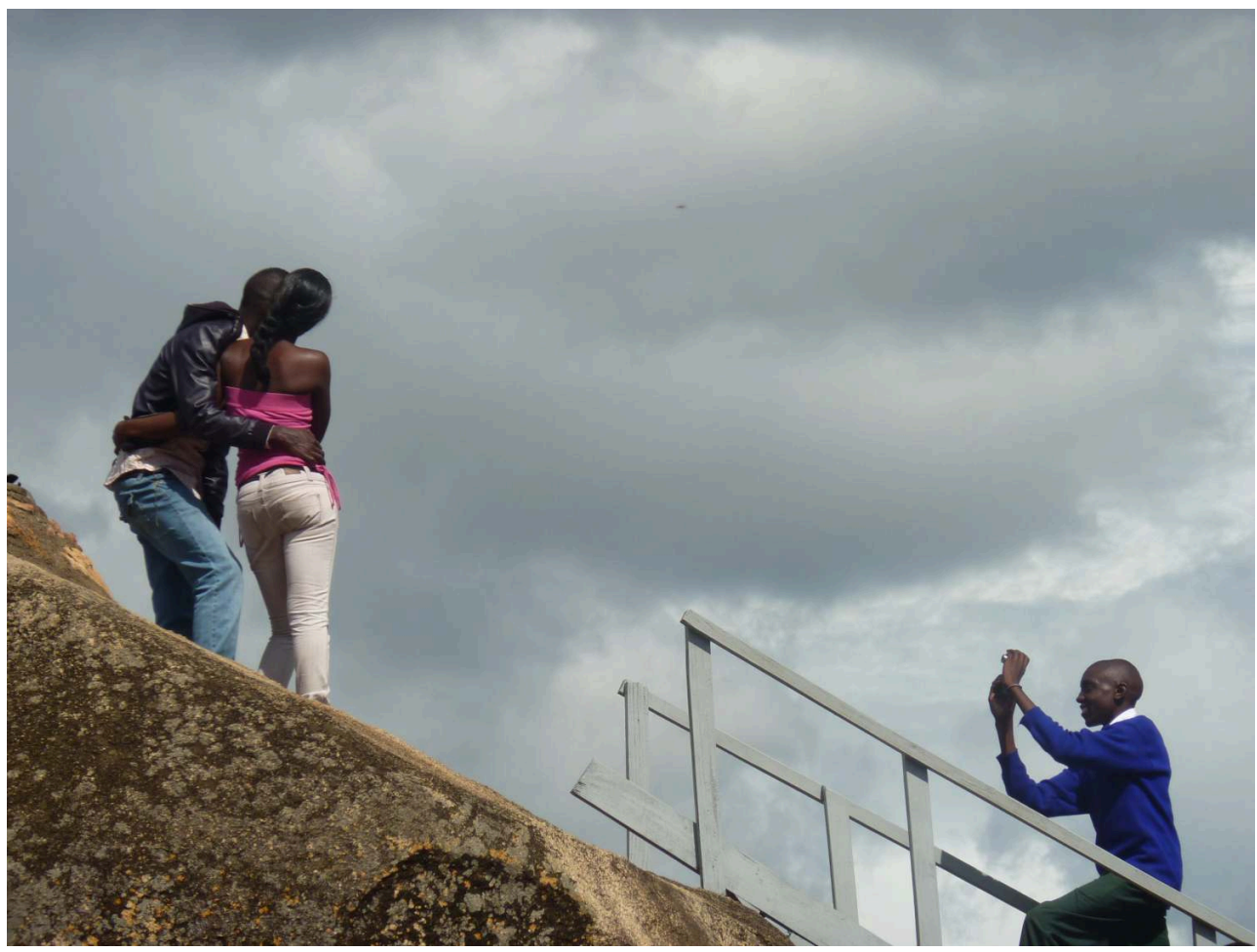

Photo : Marie-Aude Fouéré. 


\section{BIBLIOGRAPHIE}

Anderson, Benedict. 1991 [1985]. L'imaginaire national. Réflexions sur l'origine et l'essor du nationalisme. Paris : La Découverte.

Askew, Kelly. 2002. Performing the Nation: Swahili Music and Cultural Politics in Tanzania. Chicago : University of Chicago Press.

Askew, Kelly. 2006. «Sung and Unsung : Musical Reflections on Tanzanian Postsocialisms ». Africa 76 (1) : 15-43. https://doi.org/10.3366/afr.2006.0002.

Becker, Felicitas. 2013. « Remembering Nyerere: Political Rhetoric and Dissent in Contemporary Tanzania », African Affairs 112 (447) : 238-261. https://doi.org/10.1093/afra/adt019.

Bennet, Tony. 1995. The Birth of the Museum: History, Theory, Politics. London : Routledge.

Billig, Michael. 1995. Banal Nationalism. Londres : Sage Publications.

Campbell, Horace. 1992. "The Politics of Demobilization in Tanzania : Beyond Nationalism ». In Tanzania and the IMF. The Dynamics of Liberalization, dirigé par Horace Campbell et Howard Stein. Boulder : Westview Press.

Certeau, Michel de. 1980. L'invention du quotidien, 1. Arts de faire. Paris : Gallimard.

Clifford, James. 1997. «Museums as Contact Zones ». In Routes: Travel and Translation in the Late Twentieth Century, dirigé par J. Clifford, 188-219. Cambridge : Harvard University Press.

Davallon, Jean. 1999. L'Exposition à l'œuvre: Stratégies de communication et médiation symbolique. Paris : L'Harmattan.

Davallon, Jean. 1992. « Le musée est-il vraiment un média ? » Publics \& Musées, n 2 : 99-123. https://doi.org/10.3406/pumus.1992.1017.

Desvallées, André. 1976. Musées et collections publiques de France, $n^{\circ} 134$.

Merleau-Ponty, Claire et Jean-Jacques Ezrati. 2006. L'exposition. Théorie et pratique. Paris : L'Harmattan.

Edmondson, Laura. 2007. Performance and Politics in Tanzania: The Nation on Stage. Bloomington : Indiana University Press.

Fouéré, Marie-Aude, dir. 2015a. Remembering Julius Nyerere in Tanzania: History, Memory, Legacy. Dar es Salaam : Mkuki na Nyota. https://books.openedition.org/africae/643.

Fouéré, Marie-Aude. 2015b. «'Julius Nyerere': The Man, the Word, and the Order of Discourse ». In Remembering Julius Nyerere in Tanzania : History, Memory, Legacy, dirigé par Marie-Aude Fouéré, 3-32. Dar es Salaam : Mkuki na Nyota. https://books.openedition.org/africae/703.

Fouéré, Marie-Aude. 2013. « Julius Nyerere à Zanzibar. Père ou ennemi de la nation ? "Vingtième Siècle, n 118 : 61-73. https://doi.org/10.3917/ving.118.0061.

Fouéré, Marie-Aude. 2011a. « Performing and Narrating the Tanzanian Nation ». Mambo! 9 (4). https://mambo.hypotheses.org/292.

Fouéré, Marie-Aude. 2011b. « Tanzanie : la nation à l'épreuve du postsocialisme ». Politique africaine, $\mathrm{n}^{\circ} 121$ : 69-85. https://doi.org/10.3917/polaf.121.0069.

Ferguson, James. 2006. Global Shadows: Africa in the Neoliberal World Order. Durham : Duke University Press. 
Foucault, Michel. 1976. La volonté de savoir. Paris : Gallimard.

Foucault, Michel. 1997. «Il faut défendre la société». Cours au Collège de France (1975-1976). Paris : Gallimard/Seuil.

Gaugue, Anne. 1999. " Musées et colonisation en Afrique tropicale ", Cahiers d'études africaines, $\mathrm{n}^{\circ}$ 155-156 : 727-745. https://doi.org/10.3406/cea.1999.1775.

Gaugue, Anne. 1997. Les États africains et leurs musées. La mise en scène de la Nation. Paris : L'Harmattan.

Goulemot, J.-M. 2003. « De la lecture comme production de sens ». In Pratiques de la lecture, dirigé par Roger Chartier, 115-127. Paris : Payot \& Rivages.

Hooper-Greenhill, Ellean. 1992. Museums and the Shaping of Knowledge. London : Routledge.

Hunter, Emma. 2008. « Revisiting Ujamaa: Political Legitimacy and the Construction of Community in Post-Colonial Tanzania ». Journal of Eastern African Studies 2(3) : 471-485. https:// doi.org/10.1080/17531050802401858.

Kaplan, Flore E. 1994. Museums and the Making of 'Ourselves' : The Role of Objects in National Identity. London : Leicester University Press.

Languille, Sonia. 2015. «Écoles secondes communautaires en Tanzanie : la bataille des notables dans le district de Lushoto ». Politique africaine, $\mathrm{n}^{\circ} 139$ : 101-121. https://doi.org/10.3917/polaf. 139.0101.

Lonsdale, John. 1981. «States and Social Processes in Africa : A Historiographical Survey ». African Studies Review 24 (2-3) : 139-225. https://doi.org/10.2307/523904.

Lonsdale, John, et Bruce Berman. 1979. « Coping with the Contradictions : The Development of the Colonial State in Kenya, 1895-1914 ». Journal of African History 20 (4) : 487-505. https://doi.org/ $10.1017 / 50021853700017503$.

Macdonald, Sharon. 1999. The Politics of Display : Museums, Science, Culture. London, New York : Routledge.

Martin, Denis-Constant. 1988. Tanzanie, l'invention d'une culture politique. Paris : Presses de la Fondation Nationale des Sciences Politiques, Karthala.

Martin, Denis-Constant. 1989. « À la quête des OPNI (objets politiques non identifiés). Comment traiter l'invention du politique ». Revue française de science politique 39 (3) : 793-815. https:// doi.org/10.3406/rfsp.1989.394448.

Mason, Rhiannon. 2004. « Review of Witcomb, Andrea, Re-Imagining the Museum: Beyond the Mausoleum ». H-Museum, H-Net Reviews, June. https://www.h-net.org/reviews/showrev.php? $\mathrm{id}=9499$ [archive].

Mason, Rhiannon. 2006. «Cultural Theory and Museum Studies ». In A Companion to Museum Studies, dirigé par Sharon Macdonald, 17-32. Malden, Oxford : Blackwell Publishing Ltd.

Mbembe, Achille. 2001. On the Postcolony. Berkeley. Los Angeles, London : University of California Press.

Mbughuni, Lawrence A. 1974. The Cultural Policy of the United Republic of Tanzania. Paris : Unesco Press.

Mercer, Claire et Jennings, Michael. 2011. «Réhabiliter les nationalismes : convivialité et conscience nationale en Tanzanie postsocialiste ». Politique africaine, $\mathrm{n}^{\circ} 121: 87-106$. https:// doi.org/10.3917/polaf.121.0087 
Mitchell, Timothy. 1991. « The Limits of the State : Beyond Statist Approach and their Limits ». The American Political Science Review 85 (1) : 77-96. https://doi.org/10.2307/1962879.

Paget, Dan. 2020. « Again, Making Tanzania Great : Magufuli's Restorationist Developmental Nationalism », Democratization 27 (7) : 1240-1260. https://doi.org/10.1080/13510347.2020.1779223.

Phillips, Ruth B. 2005. « Re-placing Objects : Historical Practices for the Second Museum Age », The Canadian Historical Review 86 (1) : 83-110. https://doi.org/10.1353/can.2005.0086.

Poulot, Dominique. 2009. Musées et muséologie. Paris : La Découverte.

Ranger, Terence. 1971. « The 'New Historiography' in Dar es Salaam : An Answer ». African Affairs 7 (278) : 50-61. https://doi.org/10.1093/oxfordjournals.afraf.a096084.

Renan, Ernest. 1997 [1882]. Qu'est-ce qu'une nation ? Paris : Mille et Une Nuits.

Roy, Mathieu et Charles Mnyampala. 2010. «Vénération, scepticisme et rage : éclatement de la représentation de la nation dans la littérature swahilophone tanzanienne (1968-2008)». https:// halshs.archives-ouvertes.fr/halshs-00481083v1.

Ruhumbika, Gabriel. 1992. Miradi bubu ya wazalendo (Les entreprises invisibles des patriotes). Dar es Salaam : Tanzania Publishing House.

Smith, Étienne. 2013. «'Senghor voulait qu'on soit tous des Senghor' : parcours nostalgiques d'une génération de lettrés ». Vingtième Siècle, nº 118 : 87-100. https://di.org/10.3917/vin. 118.0087 .

Smith, William E. 2011 [1972]. Nyerere of Tanzania, The First Decade 1961-1971. Harare : African Publishing Group.

Stöger-Eising, Viktoria. 2000. « 'Ujamaa' Revisited: Indigenous and European Influences in Nyerere's Social and Political Thought ». Africa 70 (1) : 118-143. https://doi.org/10.3366/afr. 2000.70.1.118.

Thiesse, Anne-Marie. 1999. La création des identités nationales. Paris : Éditions du Seuil.

Witcomb, Andrea. 2003. Re-Imagining the Museum : Beyond the Mausoleum. London : Routledge.

\section{NOTES}

1. Mwalimu, surnom attribué à Julius K. Nyerere, signifie le « maître d'école » en swahili.

2. Citations tirées de questionnaires et entretiens avec le public, 14 octobre 2012. Cet article résulte de terrains au musée Nyerere en octobre 2010, 2011, 2012 et 2020 lors des journées de célébrations du décès de Nyerere.

3. Le Tanganyika, ancienne colonie britannique, obtient une indépendance négociée le 9 décembre 1961. La Tanzanie, officiellement nommée République unie de Tanzanie, naît le 26 avril 1964 d'une union du Tanganyika et de Zanzibar, protectorat britannique qui obtient son indépendance le 10 décembre 1963.

4. «Le culte des ancêtres est de tous le plus légitime; les ancêtres nous ont fait ce que nous sommes. Un passé héroïque, des grands hommes, de la gloire (j'entends de la véritable), voilà le capital social sur lequel on assied une idée nationale » (Renan, 1997 [1882] : 31).

5. Sur l'outil muséal dans la mise en scène de la nation en Afrique après les indépendances, se référer à A. Gaugue (1997). Sur la production du nationalisme par les musées, se référer aussi à $F$. Kaplan (1994).

6. Sur la construction contemporaine de Nyerere en "père de la nation ", voir M.-A. Fouéré (2015a; 2015b). 
7. Le parti qui parvient au pouvoir à l'indépendance du Tanganyika, et devient parti unique, est la TANU (Tanganyika African National Union) ; il devient le Chama Cha Mapinduzi (CCM) en 1977 après sa fusion avec le parti unique à Zanzibar, l'Afro-Shirazi Party (ASP).

8. Le public scolaire constitue le gros des visiteurs de musée en Afrique de l'Est, plus que les visiteurs privés.

9. D.-C. Martin (1998) a bien montré la place des langages politiques pour dire le politique en Tanzanie socialiste, dans l'allégeance ou la contestation. Voir aussi E. Hunter (2008).

10. Pour l'application de ce paradigme de la collaboration et de la convivialité au cas de la Tanzanie, voir L. Edmondson (2007) et K. Askew (2002).

11. Voir aussi, pour une même approche foucaldienne, E. Hooper-Greenhill (1992).

12. Voir la définition du musée par l'ICOM (International Council of Museums), une institution qui " présente un ensemble de biens culturels à des fins de conservation, d'étude, d'éducation et de délectation".

13. Voir aussi B. Anderson (1991 [1985]) et A.-M. Thiesse (1999) qui montrent comment les musées, aux côtés de l'imprimé (journal, romans), des histoires nationales ou des monuments historiques, comptent parmi les instruments de production de l'idée moderne de nation.

14. Voir R. Mason (2004) qui montre que, chez T. Bennett, « les visiteurs sont souvent ignorés, ou leurs réponses simplifiées à l'extrême (alors que) les visiteurs ne viennent pas dans les musées totalement passifs ou comme des ardoises vierges » (p. 25).

15. M. de Certeau (1980) parle des «procédés muets » par lesquels les individus se réapproprient, voire déplacent, le sens initialement voulu.

16. Parmi les représentants de cette perspective optimiste de l'institution muséale de la seconde vague de la «nouvelle muséologie », dans les années 2000, voir S. Macdonald (1999), A. Witcomb (2003), R. Phillips (2005) ou encore R. Mason (2006). Dans des approches associées au cultural et postcolonial studies, le musée est conçu comme un «espace perméable de la rencontre transculturelle " (Mason, $2006: 25$ ) où différentes voix pourraient dialoguer sur un pied d'égalité. 17. Je parle ici de hors-musée comme on parle de hors-texte pour désigner, dans l'analyse des pratiques de la lecture, les dispositions et compétences qui rendent possible la constitution d'un sens autre que le sens déposé dans le texte par son auteur. Voir J.-M. Goulemot (2003).

18. National Museum of Tanzania Act du 2 février 1980 (qui remplace le National Museum Act de 1962).

19. Toutes les traductions du swahili au français sont de l'auteure.

20. Soit 71 cents d'euros, 24 cents et 2,60 euros respectivement.

21. Citation tirée du roman de Gabriel Ruhumbika (1992), cité dans M. Roy et C. Mnyampala (2010).

22. Le groupe, proche du parti au pouvoir, a sorti trois chansons dédiées à Nyerere, préparées avant son décès et disponibles à l'achat quelques heures après l'annonce de son décès. Voir F. Becker (2013) qui retranscrit et traduit les paroles d'une des chansons de Remmy Ongala entendue en boucle pendant la période de deuil national (p. 244), « Repose en paix, Mwalimu ».

23. Nyerere est l'auteur d'une abondante littérature sur le socialisme africain (Ujamaa) qui sert de matrice à la voie de développement que le pays va poursuivre à partir de 1967. Il a aussi développé ses conceptions du pouvoir politique et de la nation dans nombre de discours publics, récemment publiés. Sur la « philosophie politique » de Nyerere, voir notamment V. Stöger-Eising (2000).

24. L'homme porte la moustache taillée comme celle longtemps portée par Nyerere, large et courte; il semble chérir une diction posée qui rappelle celle de Nyerere (observation en 2010 et 2012). Voir des observations similaires sur le mimétisme dans le cas de la mémoire du président Senghor chez les générations de lettrés sénégalais par E. Smith (2013).

25. Le musée de la Déclaration d'Arusha, situé dans la ville d'Arusha, propose des collections liées aux mouvements anticoloniaux, à l'octroi de l'indépendance et à l'entrée du pays dans l'Ujamaa. 
26. Discours de Julius Nyerere à la Tanganyika Legislative Assembly, 22 octobre 1959.

27. Ces statistiques, faites annuellement par le musée, m'ont été gracieusement mises à disposition.

28. J'ai initialement sympathisé avec Consolata (pseudonyme) à l'hôtel où nous résidions toutes les deux à Musoma, la veille des commémorations, et elle a accepté que je l'accompagne dans sa visite à Butiama.

29. Pour des évocations sur la vie spirituelle de Nyerere, voir sa biographie par W.E. Smith (2011 [1972]).

30. Le corpus de commentaires du livre d'or du musée que j'ai constitué couvre quatre ans et demi, allant de début 2008 à la mi-octobre 2012.

31. Voir Mercer et Jennings (2011) qui, contre ce qu'ils qualifient d'une «lecture influente, beaucoup plus sombre de la convivialité comme commandement proposée par Achille Mbembe ", citent des travaux soulignant que le nationalisme peut être le produit de « relations complices et interdépendances » entre dirigeants et dirigés, comme en Tanzanie (p. 91-92).

\section{RÉSUMÉS}

Le musée Julius Kambarage Nyerere en Tanzanie est un musée patriotique : l'œuvre politique nyérériste et l'histoire de la nation tanzanienne n'y font qu'un. Partant de l'association classique entre le culte des grands hommes politiques et les efforts de légitimation voire d'hégémonie politique, l'article se penche essentiellement sur la réception du musée par ses visiteurs à partir de parcours de visite et des livres d'or du musée. Ce faisant, il utilise le musée Nyerere comme un observatoire des compositions et recompositions dans les imaginaires des rapports entre gouvernants et gouvernés en Tanzanie contemporaine. Il questionne en outre la valeur heuristique du musée comme objet pour les sciences sociales.

\section{INDEX}

Index géographique : Tanzania | Tanzanie

Mots-clés : Butiama, commémorations, Julius Nyerere, musée, nation

\section{AUTEUR}

\section{MARIE-AUDE FOUÉRÉ}

IFRA-Nairobi ; Institut des mondes africains - École des hautes études en sciences sociales.

https://orcid.org/0000-0002-7565-4231 